\title{
MOTIVATION as a fundamental tool in specialized and community rehabilitation: from task-oriented training up to self recovery
}

\begin{abstract}
Alessandro Giustini
Surely in this period Europe, and some other regions in the world, has many deep economical and social criticisms, but Health Services, as built in the past century in our Countries must be defended and maintained, especially now in relation to the economical and demographical changes to support the most and enlarging frails people groups.

Rehabilitation has a specific role in maintaining Health for All : In our medical field mainly than others scientific knowledges are strongly connected with etical mission.

PRM Ph. has a leading responsibility, together with other professionals and bio-engineer in all fields in rehabilitation, with the aim to better define all the features of clinical and research pathways, appropriateness and efficacy/effectiveness, and finally outcomes measures and concrete advantages in quality of Health for patients.

So we must face in time these critical points to find a good and sustainable solution, finding alliances, promoting attention and awareness at any level.

Researches and Technologies in one hand, changes in demography and epidemiology in another hand, in the same time the efficacy/effectiveness of health interventions (from ICU up to rehabilitation and long term cares) are creating a so rapid increase of numbers and duration for rehabilitation treatments.

This Landscape opens many risks for any National Health Service, regarding globality, sustainability, accessibility needed to be guaranteed to any patient in relation to their health right .

For example the field of new technologies have high costs itself, and open some different sources for other costs if well applied :

- time for Specialists and other Professionals to know, to apply, to make very often a personalized setting for any care programs, to verify and collect results,

- rapid innovation asking change of apparatus ( change education and so on ),

- enlarging duration, number, types of treatments and follow-up for in-patients and for out-patients too (tele-rehabilitation could be not a simplifier but probably a multiplayer of needs ?).

Probably it is totally not possible to hope to follow and apply (neither in richest countries ) these developments only by methodologies/technologies/professionals working "around" (in- or out- ) patients, and in the same time it is surely not suitable to the true value of "recovery" in the people life.

On the contrary Rehabilitation Medicine must be the vay to re-acquire the people's body function, by latent powers which we have in any part of the body and mainly in the brain.

Working actively ( motivation ) to reach the best personal activity and participation must occupy a big portion of the Rehabilitation Medicine, and the process of activating the work of brain by the latent powers ( hope, whish, joy, fear, emulation ....).

As a matter of fact these are the main contents in Community Rehabilitation too, as United Nations defined in their guidelines for less developed Countries to face many aspects of disability.
\end{abstract}

